\title{
Quantification de la biomasse aérienne des palétuviers du genre Rhizophora au Delta du Saloum (Sénégal)
}

\author{
Ngor NDOUR $^{1 *}$, Bienvenu SAMBOU ${ }^{2}$ et Sara Danièle DIENG ${ }^{3}$ \\ ${ }^{1}$ Université Assane SECK de Ziguinchor, Ziguinchor, Sénégal. \\ ${ }^{2}$ Université Cheikh Anta Diop, Institut des Sciences de l'Environnement, Dakar, Sénégal. \\ ${ }^{3}$ Université Cheikh Anta Diop, Institut des Sciences de l'Environnement, UCAD, Dakar, Sénégal. \\ *Auteur correspondant, E-mail : ngorndour@univ-zig.sn; Tél : + 2215533203
}

\section{RESUME}

L'étude de la biomasse aérienne des palétuviers a fait l'objet d'un inventaire dans quatre unités d'échantillonnage. Quinze parcelles permanentes ont aussi permis d'étudier les effets de certains facteurs du milieu sur la biomasse. Dans les strates de hauteur moyenne, la biomasse sèche des racines aériennes la plus élevée est de 67 tonnes à l'hectare chez $R$. mangle. Dans les strates hautes, celle la plus élevée est de 83,3 tonnes à l'hectare. Ces deux grandes quantités de biomasse racinaire supportent aussi les biomasses sèches de tiges et autres parties les plus élevées dans la zone. En termes de comparaison, le pic de la biomasse sèche des tiges et autres parties est observé chez $R$. mangle. Quant à la biomasse racinaire sèche, le pic est noté chez $R$. racemosa/harisonnii. Dans les strates de hauteur moyenne, la biomasse sèche des tiges et autres parties est en général plus élevée que celle des racines. Dans les strates hautes, ces rapports de biomasse sont inversés. L'analyse de corrélation entre la biomasse racinaire et les facteurs étudiés montre que l'augmentation de celleci est plus liée à la hauteur de submersion qu'au type de sol. Cette augmentation serait le résultat d'une adaptation morphologique des palétuviers aux vasières meubles et à leur submersion.

() 2014 International Formulae Group. All rights reserved.

Mots clés : Biomasse sèche, strates, milieu, racinaire, tiges, autres parties.

\section{INTRODUCTION}

Le Delta du Saloum, situé au Sénégal, abrite une importante forêt de mangrove. Les palétuviers y sont représentés par les familles des Rhizophoraceae, Avicenniaceae et Combretaceae. Celle des Rhizophoraceae compte au moins deux espèces dont $R$. mangle L. et Rhizophora racemosa/harisonnii. La famille de Avicenniaceae est représentée par Avicennia germinans $\mathrm{L}$. et celle de Combretaceae par Conocarpus erectus L. et Laguncularia racemosa (L.) C.F. Gaertn. La biomasse aérienne produite par les palétuviers est constituée de feuilles et de brindilles, de bois et de racines échasses. Son abondance dépend des conditions environnementales marquées par un climat soudanien à tendance soudano-sahélienne au Nord, de la salinité des eaux estuariennes qui varie entre 38 et $90 \%$ et de divers autres facteurs (Marius, 1984; Baillon, 1988; Dacosta, 1992; Diouf, 1996; Cormier-Salem, 1999). Une partie de cette biomasse est transformée en matière minérale par les microorganismes lors du recyclage de 
la matière organique (FAO, 1994; Rajendran et Kathiresan, 2007; Ndour et al., 2012). Elle participe à l'enrichissement du milieu et à l'apport de nutriment pour le phytoplancton et les crustacés particulièrement. Le bois de mangrove est utilisé par les populations comme combustible et bois de services. Les racines aériennes fournissent des niches écologiques aux poissons, mollusques, petits crustacés et aux algues. Les sols initiaux de mangrove étant constitués de vases et de boues essentiellement, les palétuviers y développent de nombreuses racines échasses pour les compacter. Cette stratégie permet aux palétuviers de stabiliser leurs peuplements sur les vasières. Afin de mieux comprendre la corrélation entre la biomasse aérienne et les caractéristiques du milieu, la présente étude fournit des informations sur les palétuviers à travers les proportions des différentes parties de la biomasse aérienne. Elle cherche à montrer l'existence d'une relation d'équilibre entre les différentes parties de la biomasse aérienne des palétuviers en rapport avec les caractéristiques du milieu.

\section{MATERIEL ET METHODES}

Quatre unités d'échantillonnage sont identifiées dans le delta du Saloum en fonction de la hauteur des palétuviers (Figure 1). Dans chaque unité, des sous unités appelées strates basses de taille inférieure à 5 $\mathrm{m}$, strates de hauteur moyenne [5-7 $\mathrm{m}$ [ et strates haute de taille supérieure à $7 \mathrm{~m}$ sont délimitées sur la base d'une photo analyse.

Le choix des strates à inventorier a tenu compte de leur composition floristique, de leur physionomie, de leur étendue et de leur position par rapport au cours d'eau (bolon) le plus proche. Pour l'inventaire de la biomasse aérienne, l'orientation de chaque transect est déterminée à l'aide d'une boussole Silva, d'un calque d'interprétation et d'une carte topographique. Cependant, il a fallu tenir compte de la déclinaison magnétique qui est de 10³0’ au premier janvier 1991 sur la coupure de Sokone et de Thiès au 1/50000 (JICA, 1989). Diminuant de 5 minutes par année, la déclinaison a chuté jusqu'à $10^{\circ}$ environ. C'est pourquoi, la boussole Sylva a été réglée à chaque fois sur la nouvelle déclinaison de l'année 2000 pour une détermination correcte de l'orientation du transect. Néanmoins, la détermination de l'orientation des transects a parfois posé problème sur le terrain. Ces cas ont été minimes et assujettis à la faible qualité de certaines photographies aériennes au niveau de leurs extrémités. A cet effet, l'orientation de certains transects a été rectifiée sur le terrain. Le pourcentage de transects réorientés dans U1 est de $22,22 \%$, de $3,22 \%$ dans U2, de 19,04\% dans U3 et de 7,69\% dans U4. Le « pas » choisi lors de la progression sur un transect est variable. Chaque «pas » à faire est choisi au hasard dans une série continue de distances comprises entre 5 et 35 mètres. La distance maximale de $35 \mathrm{~m}$ a été retenue en fonction de la sous unité d'échantillonnage la moins étendue de grand axe égale à $100 \mathrm{~m}$. La distance minimale de $5 \mathrm{~m}$ a été choisie pour éviter la bordure du bolon et le rapprochement des placettes dans ce milieu difficile d'accès. L'évaluation de la biomasse a été faite dans des placettes de 2,5 m x 2,5 m le long des transects (Figure 2).

Le choix de la taille de la placette d'inventaire a tenu compte du temps nécessaire à sa délimitation et à la coupe rase des sujets à l'aide de pinces et de scies à bois. Cette dimension a été choisie également afin de pouvoir répéter un grand nombre de fois les coupes rases dans une strate sans affecter négativement les formations de mangrove. $\mathrm{Au}$ sein des placettes, les palétuviers sont recensés avant la coupe rase. Toutefois, $R$. racemosa/harisonnii ont été mises ensemble à cause de la difficulté de les distinguer sur la base des clés de détermination disponibles (Ndour et al., 2013). Ce recensement a permis de découvrir des sous unités d'échantillonnage mono spécifiques et des sous unités hétérogènes à l'intérieur des strates. L'évaluation de la biomasse en termes de «phytomasse » a concerné les tiges de diamètre supérieur ou égal à $3 \mathrm{~cm}$ à $10 \mathrm{~cm}$ de leur base et à découpe fin bout de $3 \mathrm{~cm}$. Ces découpes ont tenu compte des besoins des 
populations en bois de chauffe et de service. Des pesées à l'état frais des racines échasses, des tiges et du complexe constitué de feuilles, de brindilles, d'inflorescences et de propagules ont été effectuées (Figure 3).

Des pesées à l'état sec de ces trois types d'échantillon ont été effectuées après leur séchage à l'étuve à $70{ }^{\circ} \mathrm{C}$ pendant 72 heures. Le poids constant, obtenu à la fin du séchage a permis d'évaluer leur biomasse sèche. La quantité de matière sèche qui correspond à chacune de ces parties a été calculée en multipliant leur biomasse totale à l'état frais par un rapport moyen (R) issu de la pondération de la masse des échantillons à l'état sec par leur masse à l'état frais selon la formule suivante :

$\mathrm{Q}_{\mathrm{ms}}=\mathrm{BTF} \times \mathrm{R}$ avec $\mathrm{R}=\mathrm{BES} / \mathrm{BEF}$

$\mathbf{Q}_{\mathbf{m s}}=$ quantité de matière sèche;

$\mathrm{BTF}=$ biomasse totale à l'état frais d'une entité de l'espèce;

BES = biomasse à l'état sec d'une entité;

$\mathrm{BEF}=$ biomasse à l'état frais de la même entité.

Dans le cadre de la recherche de facteurs explicatifs de la variation de la biomasse aérienne des différentes entités, 15 parcelles permanentes ont été installées du Nord au Sud de la zone d'étude. La hauteur de submersion des palétuviers y est mesurée à l'aide d'échelles limnométriques à l'étale de pleine mer aux mois de janvier, avril et septembre. Les types de sols y sont aussi déterminés à la suite d'une analyse granulométrique et texturale au laboratoire. Ces données ont permis de montrer l'existence d'une corrélation entre la variation de la biomasse aérienne et celle des caractéristiques du milieu. Le traitement des données a tenu compte de la composition floristique des sous unités d'échantillonnage. Ceci a permis, à postériori, de regrouper les peuplements homogènes d'une part et ceux hétérogènes d'autre part. La raison du choix de ce traitement est inhérente à une meilleure prise en compte de l'hétérogénéité des strates due à la contiguïté de peuplements d'espèces différentes. Il prend également en compte la taille et l'étendue des peuplements d'espèces différentes d'une même strate. Il a permis d'évaluer la biomasse dans l'intervalle de confiance «biomasse moyenne \pm 2 fois la valeur de l'écart type par strate et par unité d'échantillonnage à l'aide des logiciels XlsatPro.6.1.9 et Excel. Pour la comparaison des différentes entités de biomasse, le degré d'humidité de chacune d'entre elles a été calculé à l'aide de la formule suivante :

$\mathrm{H}=\frac{M_{s}-M_{o}}{M_{o}} \times 100$

$\mathrm{M}_{\mathrm{s}}=$ matière saturée ou fraîche et $\mathrm{M}_{\mathrm{o}}=$ matière sèche ou anhydre

\section{RESULTATS}

Les résultats obtenus dans les quatre unités d'échantillonnage (U1, U2, U3 et U4) portent sur la biomasse aérienne des parties considérées et leur comparaison entre les différentes unités d'échantillonnage. Ils renseignent aussi sur les corrélations qui existent entre l'importance de la biomasse et certains facteurs du milieu.

\section{Les résultats de l'unité 1}

La superficie de l'unité U1 est estimée à 5400 ha. La photo-interprétation a permis d'y identifier des sous-unités d'échantillonnage constituées de mangrove haute à moyenne, de mangrove basse, de tannes et de chenaux (Figure 4).

La superficie de la mangrove haute à moyenne y est estimée à 49,6 ha soit $0,9 \%$ de la superficie de U1. Celle inventoriée est de 0,01375 ha et représente $0,027 \%$ de la superficie des peuplements de palétuviers. La biomasse à l'état frais des racines est de 82,6 tonnes à l'hectare, celle des tiges est de 77,4 et celle des feuilles et autres composantes de 57,5. Les rapports moyens (R) issus de la pondération de la masse d'échantillons à l'état sec par leur masse à l'état frais sont respectivement de 0,51 pour les racines, 0,71 pour les tiges et 0,39 pour les feuilles et autres composantes de $R$. mangle. Ainsi, la quantité de matière sèche correspondante est de 42,2 tonnes à l'hectare pour les racines, 55 tonnes pour les tiges et 22,5 tonnes pour les feuilles 
et autres composantes. Cette variation de masse de l'état frais à l'état sec est liée, entre autres facteurs, à une différence de degré d'humidité des trois parties considérées. Celui-ci est de $98,2 \%$ pour les racines, de $43,3 \%$ pour les tiges et de $179,3 \%$ pour les feuilles et autres composantes.

Dans les parcelles permanentes de U1, les sols initiaux de mangrove sont limoneux fins associés à des sables fins à grossiers. La hauteur de submersion moyenne y est de $18,76 \mathrm{~cm}$ avec des pics de $45 \mathrm{~cm}$ et de faibles hauteurs d'eau de $2,8 \mathrm{~cm}$ à l'étale de pleine mer.

\section{Résultats de l'unité 2}

Les sous unités de U2 sont essentiellement constituées de mangrove haute à moyenne, de mangrove basse, de tannes relativement étendues, de chenaux, de passes, de cordons sableux et d'amas coquilliers (Figure 5). La superficie totale de U2 est estimée à 6604,9 ha. La superficie de la mangrove haute à moyenne confondues est de 97,8 ha, soit $1,5 \%$ de la superficie de U2.

La superficie inventoriée dans U2 est de 0,02375 ha. Elle représente $0,024 \%$ des strates de mangrove haute à moyenne de l'unité. $65,7 \%$ de cette superficie a été inventoriée dans les peuplements purs de $R$. mangle, $23,7 \%$ dans ceux purs de $R$. racemosa/harisonnii et 10,6\% dans les zones de contact. Le rapport de pondération entre la masse à l'état sec et la masse à l'état frais des échantillons a donné pour les racines de $R$. mangle $0,56,0,73$ pour les tiges et 0,41 pour les feuilles et les autres composantes. Ce rapport est pour les racines de $R$. racemosa/harisonnii de 0,50 , celui des tiges de 0,65 et celui des feuilles et autres composantes de 0,38 .

Dans les peuplements de mangrove moyenne à $R$. mangle, la biomasse à l'état frais des racines est de 94,9 tonnes à l'hectare, celle des tiges de 61,5 et celle des feuilles et autres composantes de 59,8. La quantité de matière sèche correspondante est de 53,2 tonnes à l'hectare pour les racines, 44,8 pour les tiges et 24,5 pour les feuilles et autres composantes.

Dans les peuplements de mangrove moyenne à $R$. racemosa/harisonnii, la biomasse à l'état frais des racines est de 122,7 tonnes à l'hectare, celle des tiges de 66,6 et celle des feuilles et autres éléments de 39,02. La quantité de matière sèche correspondante est de 61,3 tonnes à l'hectare pour les racines, 43,3 pour les tiges et 14,8 pour les feuilles et autres composantes.

Dans les zones de contact de peuplements d'espèce différentes, la biomasse à l'état frais des racines est de 79,2 tonnes à l'hectare, celle des tiges de 54,2 et celle des feuilles et autres composantes de 58,2.

Le degré d'humidité des racines de $R$. mangle est de $78,57 \%$, celui des tiges de $36,9 \%$ et celui des feuilles et autres composantes $143,90 \%$. Il est de $100 \%$ pour les racines, de 53,8\% pour les tiges et de $163,2 \%$ pour les feuilles et autres composantes de $R$. racemosa/harisonnii.

Dans les parcelles permanentes de U2, les sols initiaux de mangrove sont limonosableux avec des proportions assez importantes de sables fins. La hauteur de submersion moyenne des vasières y est de $31,5 \mathrm{~cm}$ à l'étale de pleine mer.

\section{Résultats de l'unité 3}

Dans U3, les sous unités sont constituées de mangrove haute, de mangrove moyenne, de mangrove basse, de tannes, de chenaux, d'îles et d'îlots à amas coquilliers (Figure 6).

La superficie totale de U3 est estimée à 3000 ha. La mangrove haute à moyenne occupe 197,9 ha, soit $6,6 \%$ de l'unité. La superficie totale inventoriée est de 0,039375 ha. Elle représente $0,02 \%$ de la superficie des strates identifiées dans U3. Les rapports moyens, entre la masse de matière sèche de chaque partie et sa masse de matière fraîche, sont respectivement de 0,56 pour les racines, de 0,70 pour les tiges et de 0,40 pour les feuilles et autres composantes de $R$. mangle. Concernant $R$. racemosa/harisonnii, ces rapports sont de 0,52 pour les racines, de 0,69 
pour les tiges et de 0,42 pour les feuilles et autres composantes.

Dans les peuplements de mangrove haute à $R$. racemosa/harisonnii, la superficie inventoriée est de 0,0175 ha. Elle représente $44,4 \%$ de la superficie inventoriée. Dans ces peuplements, la biomasse à l'état frais des racines est de 157,2 tonnes à l'hectare, celle des tiges est de 95,5 et celle des feuilles et autres composantes de 38,2. La quantité de matière sèche correspondante est de 83,3 tonnes à l'hectare pour les racines, 66 pour les tiges et 15,3 pour les feuilles et les autres composantes.

Dans les peuplements de mangrove moyenne à $R$. racemosa/harisonnii, la superficie inventoriée est de 0,006875 ha et représente $17,4 \%$ de la superficie inventoriée dans l'unité. Dans ces peuplements, la biomasse à l'état frais des racines est de 108,5 tonnes à l'hectare, celle des tiges de 21,9 et celle des feuilles et autres composantes de 46,6. La quantité de matière sèche correspondante est de 57,5 tonnes à l'hectare pour les racines, 15,2 pour les tiges et 18,6 pour les feuilles et autres composantes.

Dans les peuplements de mangrove moyenne à $R$. mangle, la superficie inventoriée est de 0,010625 ha. Elle représente $27 \%$ de la superficie inventoriée dans cette unité. La biomasse à l'état frais de ces peuplements est de 103 tonnes à l'hectare pour les racines, celle des tiges de 37,2 et celle des feuilles et autres composantes de 40,8. La quantité de matière sèche correspondante est de 57,7 tonnes à l'hectare pour les racines, 26 pour les tiges et 16,3 pour les feuilles et autres composantes.

Dans les zones de contacts, la superficie inventoriée est de 0,004375 ha. Elle représente $11,1 \%$ de la superficie inventoriée dans l'unité. La biomasse à l'état frais des racines est de 104,5 tonnes à l'hectare, celle des tiges est de 52,1 et celle des feuilles et autres composantes de 33,4.

Le degré d'humidité des racines est de $78,6 \%$, de $42,9 \%$ pour les tiges et de $150 \%$ pour les feuilles et autres composantes de $R$. mangle. Il est de $88,7 \%$ pour les racines, de $44,9 \%$ pour les tiges et de $150 \%$ pour les feuilles et autres composantes des strates de hauteur moyenne de $R$. racemosa/harisonnii. Pour les strates hautes de la même espèce, ce taux est de $88,7 \%$ pour les racines, de $49,9 \%$ pour les tiges et de $150 \%$ pour les feuilles et autres composantes.

Dans les parcelles permanentes de U3, les sols initiaux de mangrove sont limonosablo-argileux dans les strates hautes et limono-sableux dans les strates moyennes à $R$. mangle ou à $R$. racemosa/harisonnii. La hauteur moyenne de submersion est de 67,06 $\mathrm{cm}$ dans les strates hautes à $R$. racemosa/harrisonnii et de $52 \mathrm{~cm}$ dans les peuplements de $R$. mangle. En moyenne, la hauteur de submersion est de 59,80 cm dans U3.

\section{Résultats de l'unité 4}

Les strates identifiées dans U4 sont constituées de mangrove haute, de mangrove moyenne, de mangrove basse, de tannes, de chenaux, de passes, etc. (Figure 7). La superficie totale de U4 est de 1850 ha. Celle de la mangrove haute et de la mangrove de hauteur moyenne est de 149,4 ha représentant $8 \%$ de la superficie totale. Les tannes sont estimées à 106 ha soit 5,8\% de la superficie de l'unité. $\mathrm{Au}$ plan floristique, $R$. racemosa/harisonnii, $R$. mangle, Laguncularia racemosa et Avicennia germinans ont été rencontrées. $R$. mangle et $R$. racemosa/harisonnii forment généralement des peuplements purs. Toutefois, des zones de contacts existent entre ces deux espèces.

La superficie totale inventoriée est de 0,030625 ha dans U4. Elle représente 0,020\% de l'ensemble de ses strates. Les rapports moyens entre la masse de matière sèche de chaque partie et sa masse de matière fraîche, sont respectivement de 0,55 pour les racines, de 0,68 pour les tiges et de 0,41 pour les feuilles et composantes de $R$. mangle. Concernant $R$. racemosa/harisonnii, ces 
rapports sont de 0,53 pour les racines, de 0,67 pour les tiges et de 0,37 pour les feuilles et autres composantes.

Dans les peuplements de mangrove haute à $R$. racemosa/harisonnii, la biomasse à l'état frais des racines échasses est de 144,6 tonnes à l'hectare, celle des tiges est de 93,4 et celle des feuilles et autres composantes de 30,5. La biomasse de matière sèche correspondante est de 76,6 tonnes à l'hectare pour les racines, de 62,5 pour les tiges et de 11,3 pour les feuilles et autres composantes.

Dans les peuplements de mangrove moyenne à $R$. mangle, la biomasse pour chacune des parties considérées est de 121,8 tonnes à l'hectare pour les racines échasses, de 101,6 pour les tiges et de 52,7 pour les feuilles et autres composantes. La biomasse sèche des racines échasses est de 67 tonnes à l'hectare, celle des tiges est de 69,1 et celle des feuilles et autres composantes est de 21,6.

Dans les zones de contacts entre peuplements purs, la biomasse à l'état frais des racines est de 126,4 tonnes à l'hectare, celle des tiges de 29,4 et celle des feuilles et autres composantes de 23,2.

Le degré d'humidité des racines de $R$. mangle est de $81,8 \%$, celui des tiges de $47,6 \%$ et celui des feuilles et autres composantes de $134,9 \%$. Il est de $88,7 \%$ pour les racines de $R$. racemosa/harisonnii de la strate haute, de $49,3 \%$ pour les tiges et de $170,3 \%$ pour les feuilles et autres composantes.

Dans les parcelles permanentes de U4, les sols initiaux de mangrove sont limonosableux à limoneux. La hauteur moyenne de submersion varie entre 2,2 et $114 \mathrm{~cm}$. En moyenne, elle est de $42,32 \mathrm{~cm}$ dans l'unité d'échantillonnage.

\section{Analyse comparative de la biomasse aérienne des différentes parties des palétuviers}

L'analyse de la variation de la biomasse racinaire en fonction de l'espèce, montre une différence hautement significative avec $\mathrm{p}=0,049$ (Tableau 1). Le test de Fisher indique aussi l'existence d'une plus petite différence significative pour la même modalité.

L'analyse de la variation de la biomasse racinaire en fonction de la stratification verticale (strate haute, strate de hauteur moyenne), a aussi montré une différence hautement significative avec $\mathrm{p}=$ 0,012 (Tableau 2).

Concernant la variation de la biomasse des tiges et autres composantes, en fonction des variables (espèce et strate), aucune différence significative n'a été notée par les tests de Tukey et de Fisher.

Au-delà des différences significatives notées dans les Tableaux 1 et 2, la Figure 8 montre que chez la même espèce, la biomasse racinaire ne varie pas significativement. Elle montre aussi que la biomasse racinaire est toujours légèrement plus importante que celle des autres parties quel que soit le type de strates et l'unité d'échantillonnage.

A l'état sec, la biomasse racinaire est moins importante que le cumul de la biomasse des tiges et des autres composantes de $R$. mangle dans U1. Dans U2, la situation est semblable pour la même espèce. Par contre, dans la même unité, la biomasse racinaire sèche des strates moyenne à $R$. racemosa/harisonnii est plus importante que celle cumulée des tiges et autres composantes. Dans U3, la biomasse racinaire sèche des peuplements de mangrove haute à moyenne de $R$. racemosa/harisonnii est plus importante que celle des tiges et autres parties cumulées. Dans les peuplements de mangrove moyenne à $R$. mangle, la biomasse racinaire sèche est aussi plus importante que celle des autres parties. En termes de comparaison, on note une inversion des rapports de proportionnalités des différentes parties par rapport aux résultats obtenus dans U1 et U2 pour la même espèce. Cette inversion des rapports de proportionnalités montre que la variation des différentes parties de la biomasse n'est pas seulement fonction de l'espèce et de la strate. Il y aurait donc d'autres facteurs qui contribuent à la variation 
de la biomasse des différentes parties des palétuviers dans la zone d'étude.

\section{Analyse de la variation de la biomasse en fonction des paramètres du milieu}

L'analyse de corrélation entre les différents paramètres étudiés a permis de comprendre la variation de la biomasse chez $R$. mangle et $R$. racemosa/harisonnii. Le type de sol étant quasiment identique dans les unités d'échantillonnage (dominance des limons), le test $\mathrm{Z}$ de corrélation permet de montrer que c'est la hauteur de submersion des vasières qui explique le mieux la variation de la biomasse racinaire chez le genre Rhizophora avec $\mathrm{R}=0,542$. Lorsque l'on considère l'espèce, l'analyse comparative des valeurs de biomasse montre que celles des racines sont toujours plus élevées que celles des tiges et autres parties chez $R$. racemosa/harisonnii (Figure 9).

Cette logique ne prévaut pas chez $R$. mangle qui présente des rapports de proportionnalités inversés entre le Nord (U1 et U2) et le Sud (U3) de la zone d'étude.
Considérant l'importance des limons dans les sols initiaux de mangrove du Delta du Saloum, l'analyse du graphique montre que le type de sol n'est pas fondamentalement le facteur qui explique la variation de la biomasse chez le genre Rhizophora. D'ailleurs, il a été noté chez $R$. mangle que la biomasse racinaire augmente en fonction de l'importance de la hauteur de submersion dans la zone. Dans U3 et U4 où les hauteurs de submersion sont plus importantes, les biomasses racinaires de l'espèce dépassent largement celles notées dans U1 et U2. Le constat général est que la hauteur de submersion semble plus impactée positivement la biomasse racinaire chez le genre Rhizophora au Delta du Saloum. Toutefois, ce constat n'exclue pas l'influence d'autres facteurs notamment le caractère meuble des vasières ou la compacité acquise à partir d'un certain stade de maturité de l'écosystème.

Tableau 1: Analyse de la variance de la biomasse racinaire en fonction de l'espèce

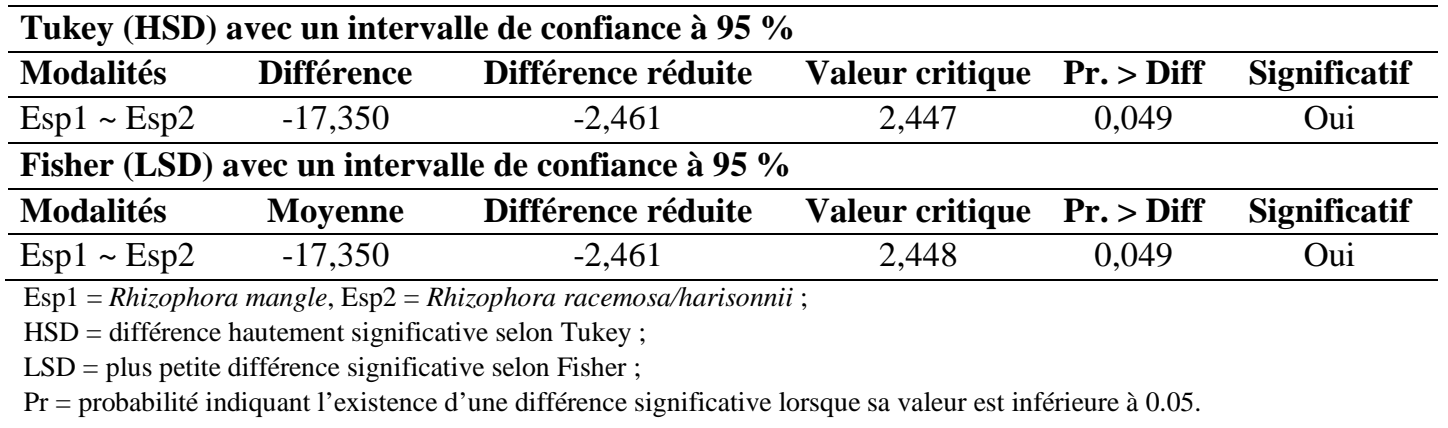

Tableau 2: Analyse de la variance de la biomasse racinaire en fonction de la strate

\begin{tabular}{|c|c|c|c|c|c|}
\hline \multicolumn{6}{|c|}{ Tukey (HSD) avec un intervalle de confiance à $95 \%$} \\
\hline Modalités & Différence & Différence réduite & Valeur critique & Pr. > Diff & Significatif \\
\hline $\mathrm{Sm} \sim \mathrm{Sh}$ & $-23,300$ & $-3,567$ & 2,447 & 0,012 & Oui \\
\hline
\end{tabular}




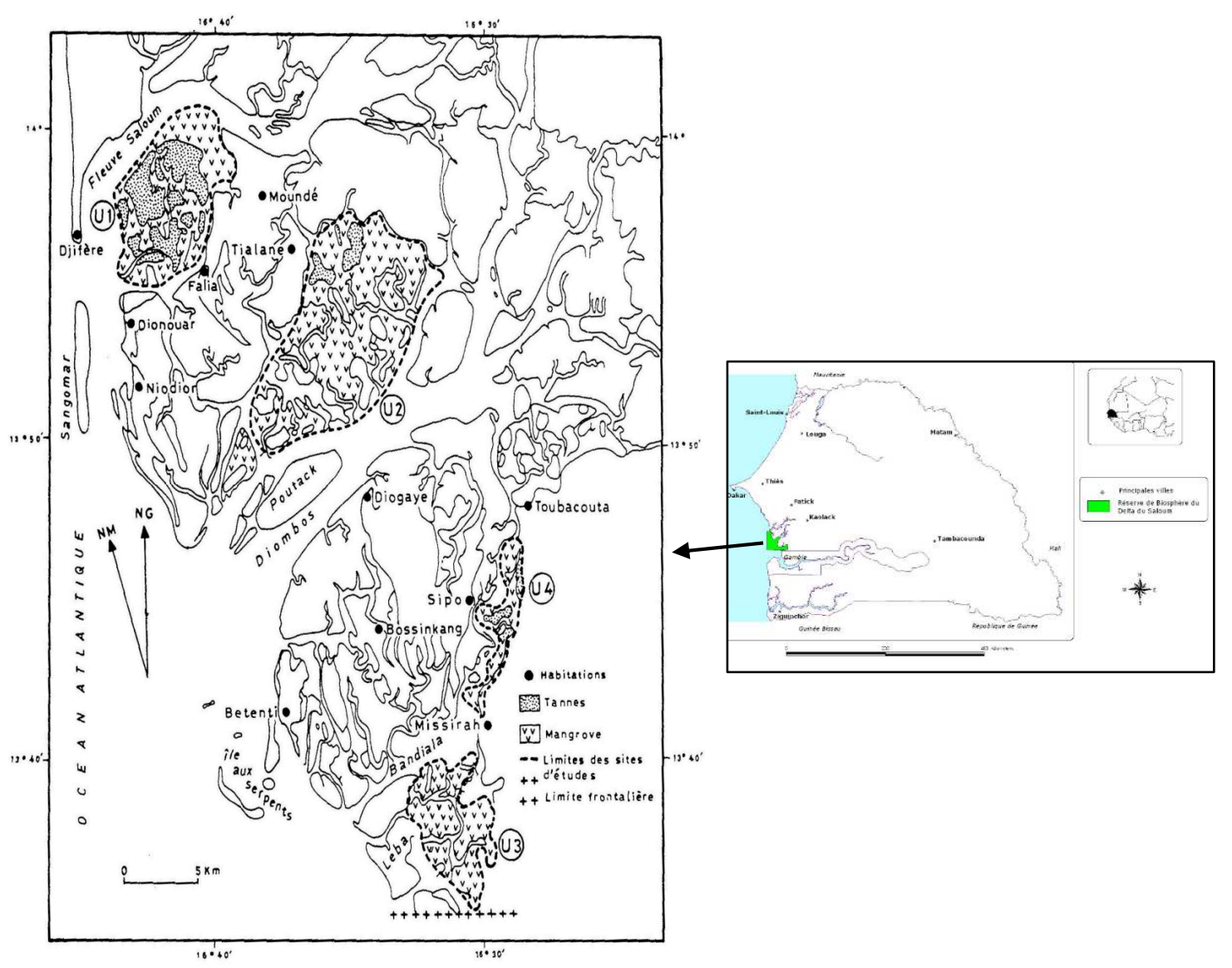

Figure 1: Localisation des unités d'échantillonnage dans le Delta du Saloum.

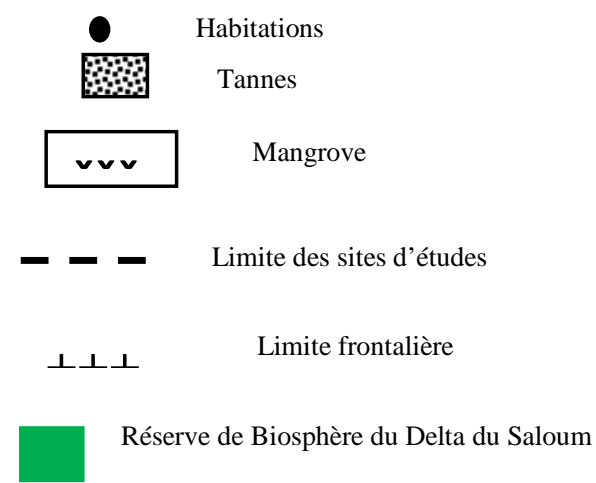




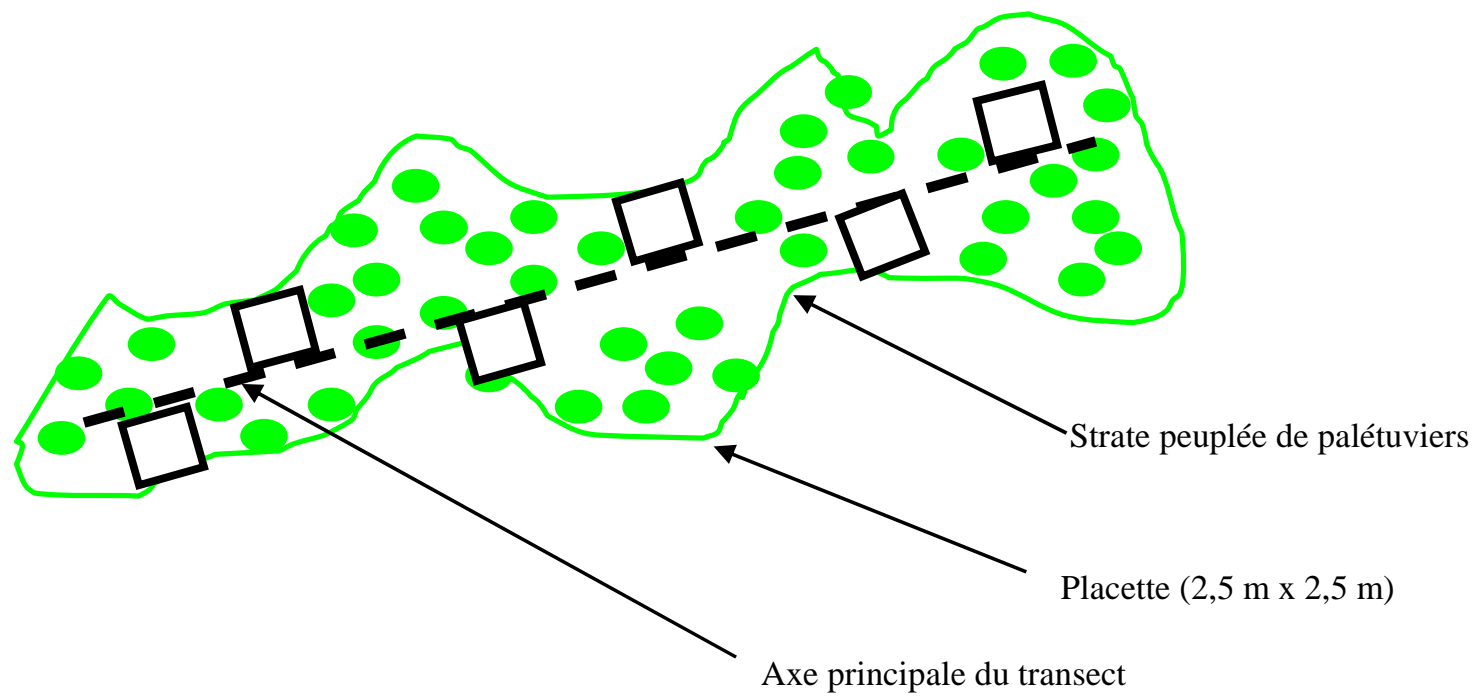

Figure 2 : Dispositif de collecte des données sur la biomasse.

Palétuviers

- Axe principale du transect
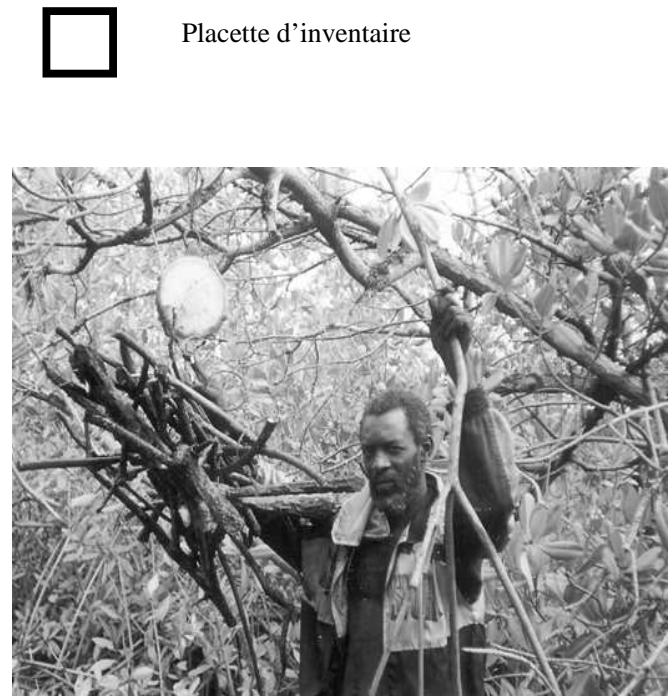

Figure 3: Pesée des racines sur le terrain. 


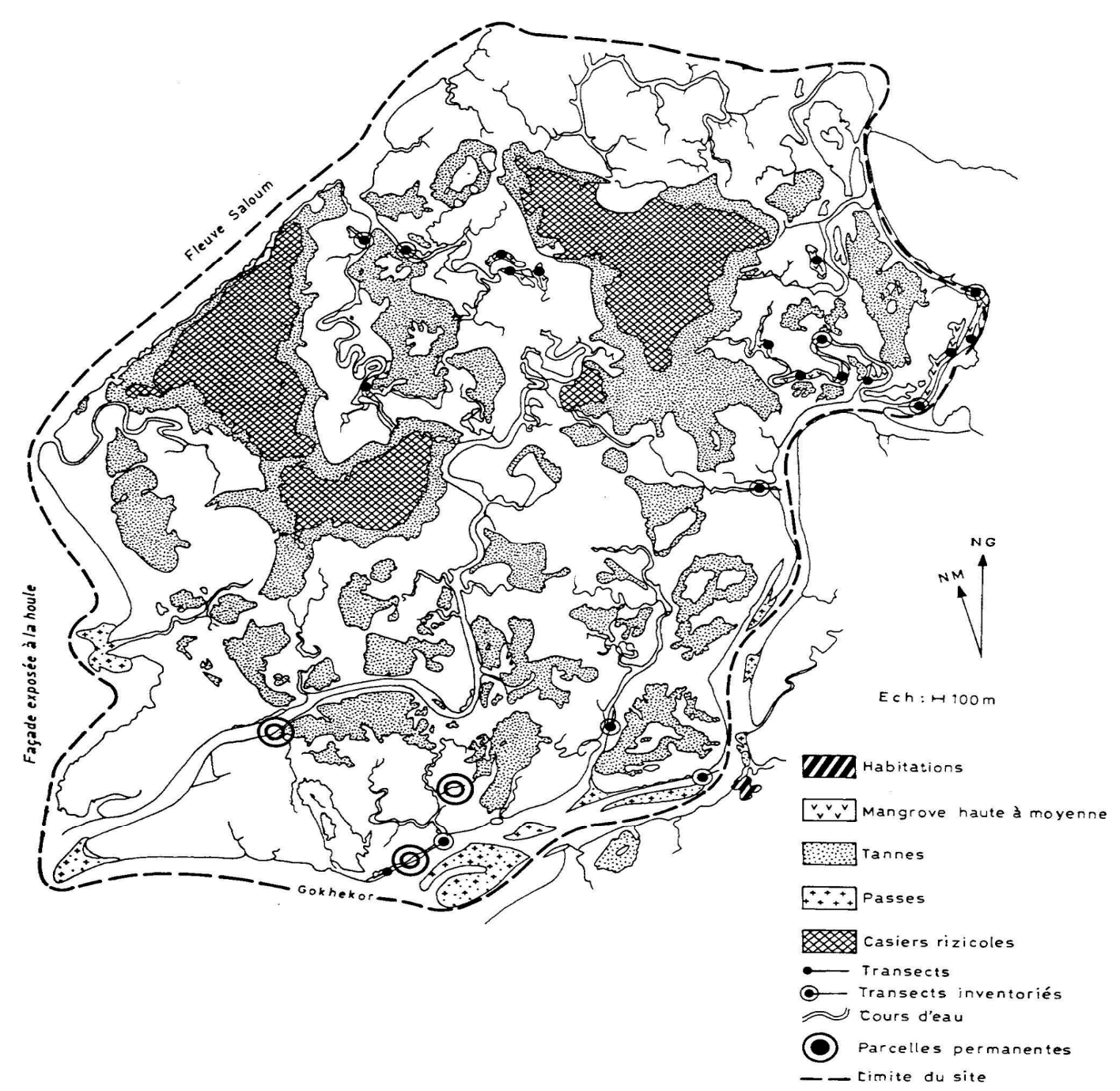

Figure 4: Occupation des sols dans l'unité 1. 


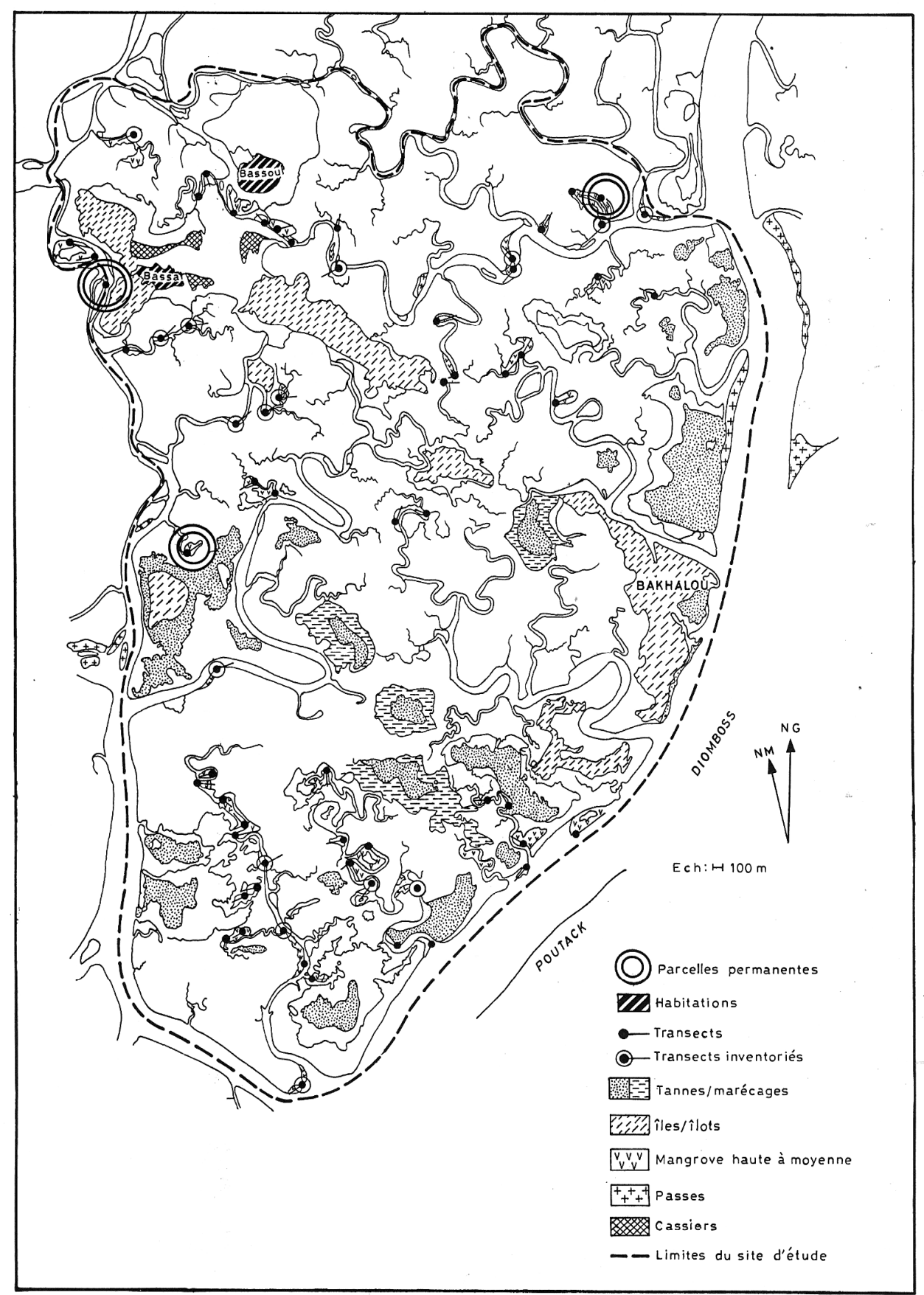

Figure 5: Carte d'occupation des sols dans l'unité 2. 


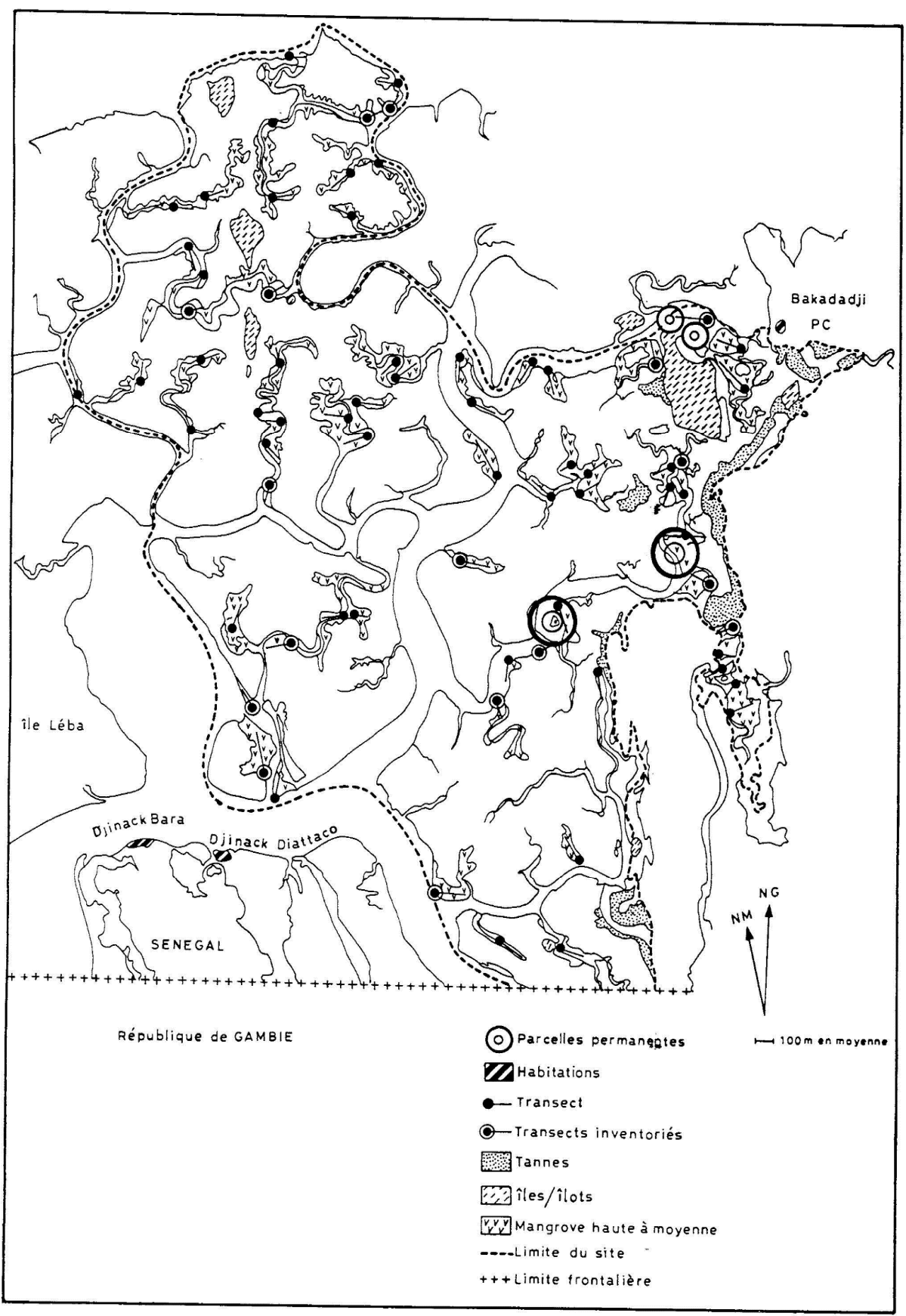

Figure 6 : Carte d'occupation des sols dans l'unité 3. 


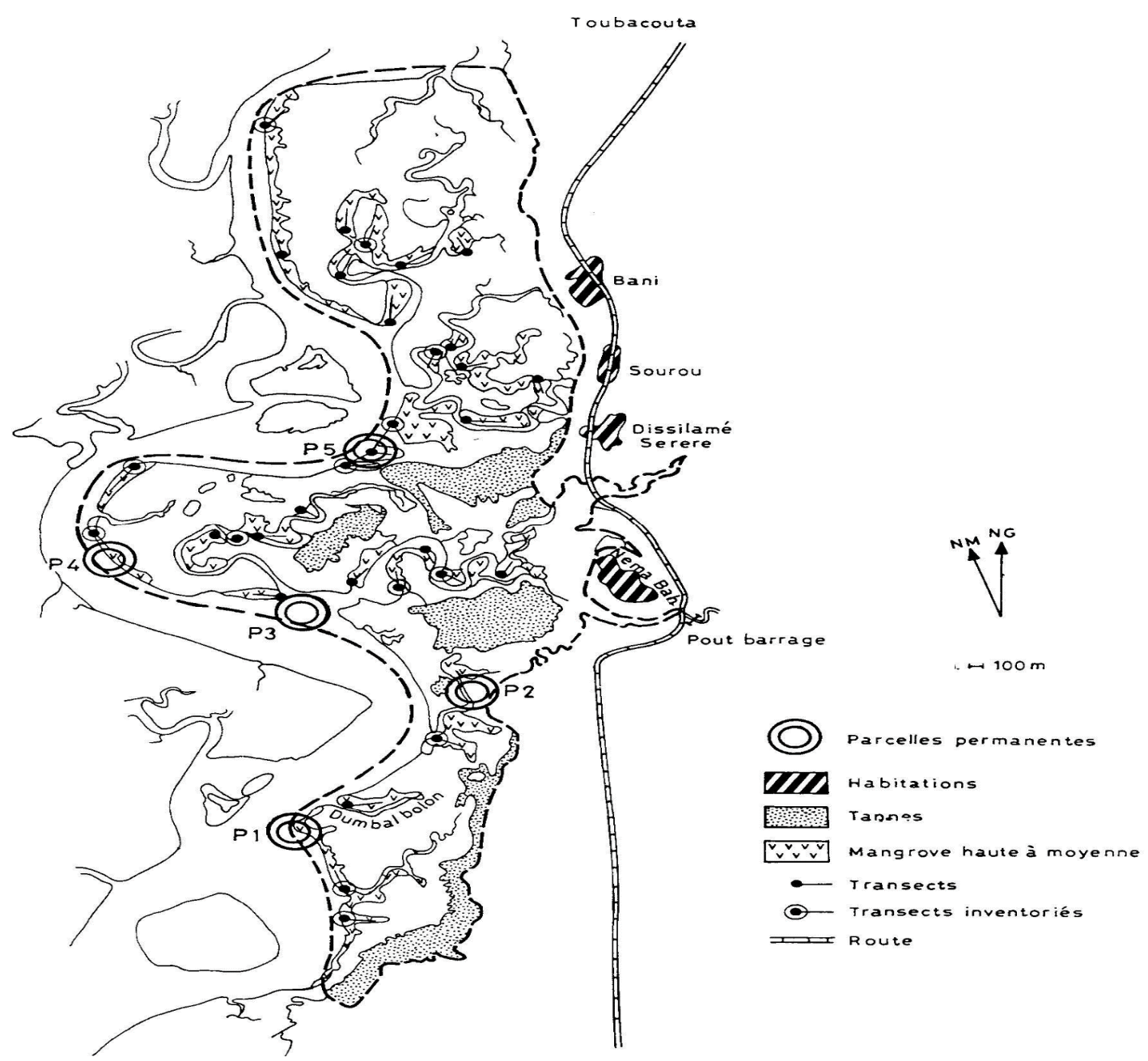

Figure 7: Carte d'occupation des sols dans l'unité 4. 


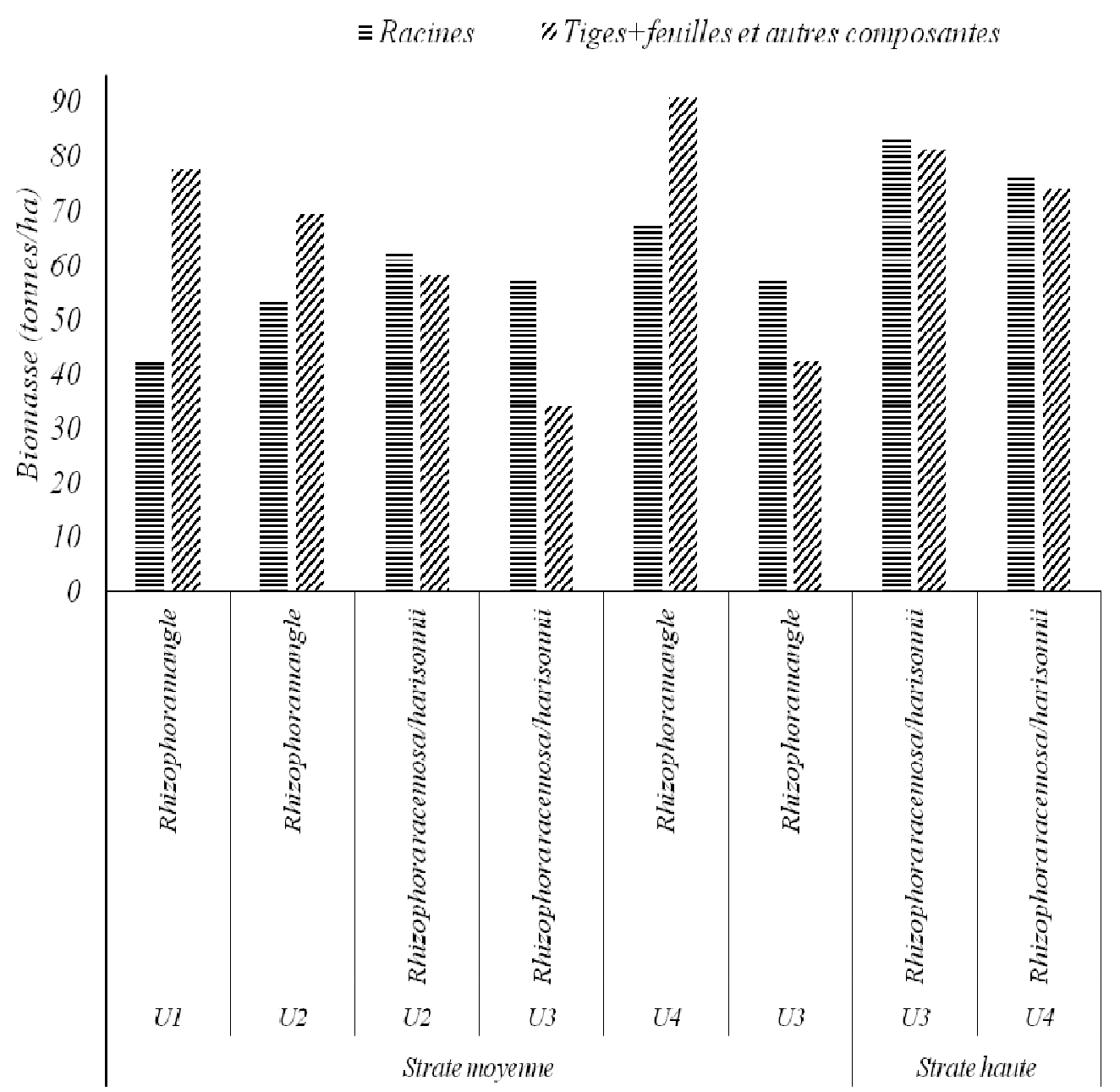

Figure 8: Comparaison de la biomasse racinaire et celle des autres parties.

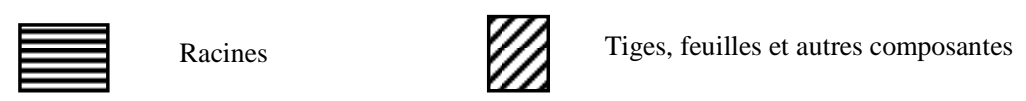




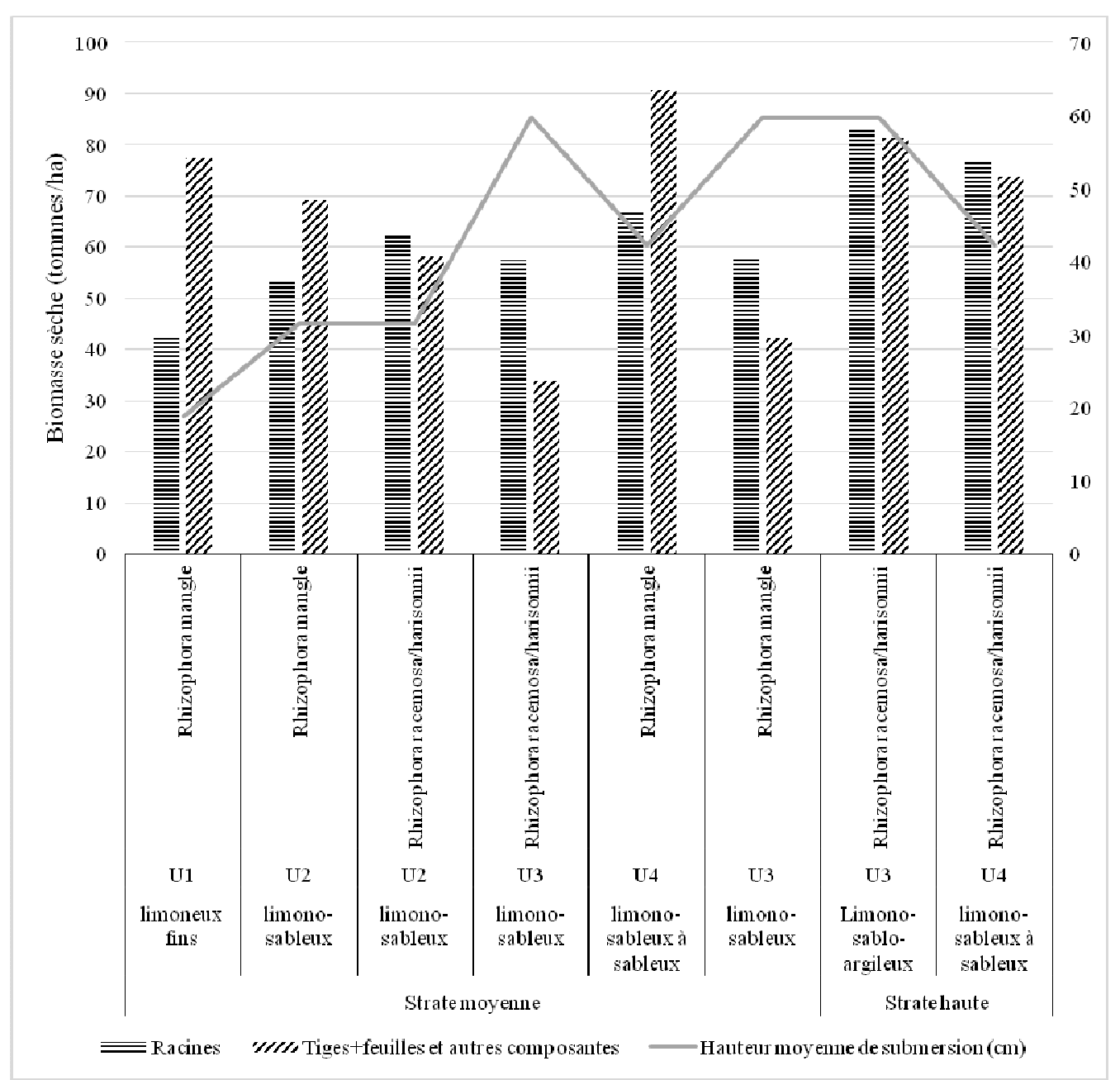

Figure 9: Variation de la biomasse aérienne en fonction du type de sol et de la hauteur de submersion.

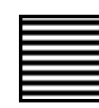

Racines,

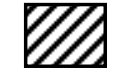

Tiges, feuilles et autres composantes,

Hauteur moyenne de submersion.

\section{DISCUSSION}

La discussion porte sur l'état de la biomasse aérienne, sa variation entre les différentes parties considérées ainsi que les corrélations qui existent entre cette variation et les facteurs édaphiques et hydrologiques du milieu.
Facteurs de conversion de la biomasse fraîche des palétuviers

L'estimation de la «phytomasse» sèche a été possible grâce à la détermination de facteurs de conversion de la biomasse fraîche en biomasse sèche pour les différentes parties (racines, tiges, feuilles et autres composantes). La comparaison de ces différents facteurs de conversion entre les unités d'échantillonnage 
montre que leur variation est faible. Le plus grand écart-type étant de 0,04 pour $R$. mangle entre les sites U1 et U2. Cependant, le facteur de chaque partie a été retenu du fait de la grande variation de masse fraîche observée et $\mathrm{du}$ souci d'analyser les résultats des différentes unités d'échantillonnage par espèce. Cette démarche s'est avérée pertinente dans la mesure où le degré d'humidité des feuilles et autres composantes a varié de $44,9 \%$ chez $R$. mangle entre U1 et U4. Cette grande variation du degré d'humidité n'estelle pas une réponse adaptative des palétuviers à la forte variabilité de la salinité des eaux dans le Delta du Saloum ? Le cas échéant, plus l'eau de l'estuaire est salée, plus élevé sera le degré d'humidité des feuilles et autres composantes, bien sûr, dans les limites de tolérance de la salinité par l'espèce. Ce raisonnement s'appuie sur l'existence dans les feuilles de Rhizophora d'organes spéciaux capables de stocker l'excès de sel jusqu'à la chute de celle-ci après la senescence (Wilmart, 1996). Dans la même logique, ce degré élevé d'humidité pourrait être lié à l'influence de la salinité comme Faye et al. (2008) l'ont démontré au Delta du Saloum.

\section{La biomasse des palétuviers du genre Rhizophora}

La biomasse sèche des racines de $R$. mangle est de 42,2 tonnes à l'hectare dans U1, de 53,2 dans U2, de 57,7 dans U3 et de 67 dans U4. Ainsi, la présente étude montre que la biomasse sèche des racines échasses de l'espèce est plus importante au Sud de la zone (U3 et U4) qu'au Nord (U1). Cette réalité pourrait s'expliquer par la variation de la microtopographie du milieu, la nature des vasières (texture, structure et compacité), leur qualité (fertilité) et l'importance de leur hauteur de submersion en marée haute. L'une de ces hypothèses est vérifiée au Saloum où les vasières du Nord sont plus élevées et plus compactes que celles du Sud (Ndour, 2005). Elles sont aussi submergées par une lame d'eau moyenne de $18,76 \mathrm{~cm}$ en marée haute, contrairement aux vasières du Sud où la colonne d'eau plus importante peut fluctuer entre 40 et $60 \mathrm{~cm}$. Cette différence de caractéristiques environnementales observées du Nord au Sud, expliquerait la variabilité de l'adaptation de $R$. mangle par rapport aux conditions du milieu. Toutefois, il ressort de l'analyse de corrélation que le type de sol n'influence pas substantiellement la variation de la biomasse racinaire. D'ailleurs, il est logique qu'une faible compacité des vasières puisse expliquer, chez $R$. mangle, le besoin de développer une structure racinaire plus forte et d'envergure pour son ancrage et sa stabilité. Par ailleurs, l'importance de la biomasse racinaire pourrait aussi s'expliquer par le nombre élevé de grosses racines chez certains sujets de gros calibres comme c'est le cas dans U3 et U4. A ce sujet, Imbert (1985) a noté que la contribution des racines aériennes et du bois fort augmente avec l'accroissement du diamètre des tiges à $1,30 \mathrm{~m}$ du sol dans la mangrove de la Guadeloupe. Les résultats obtenus au niveau du Delta du Saloum corroborent donc la variation de la biomasse racinaire en fonction du calibre des tiges qu'elles supportent.

Chez $R$. racemosa/harisonnii, appartenant à la strate moyenne, la biomasse sèche des racines est de 61,3 tonnes à l'hectare dans U2 et de 57,5 dans U3. L'étude montre que la biomasse sèche racinaire est décroissante de U2 vers U3. Cette baisse de la biomasse serait liée au fait que les peuplements de l'espèce sont généralement en bordure des bolons au niveau de U2 où la hauteur de submersion moyenne est importante en marée haute $(53,2 \mathrm{~cm})$. Dans $\mathrm{U} 3$, les peuplements de l'espèce sont souvent situés derrière les strates hautes où la hauteur d'eau est faible à l'étale de pleine mer. Dans ces conditions, $R$. racemosa/harisonnii développent des racines aériennes quasiverticales ou arcs-boutants qui supportent une faible biomasse aérienne. Ainsi, bien que la physionomie et la composition floristiques des peuplements soient la même dans les deux 
unités (U2 et U3), le besoin en racines pour l'ancrage de l'espèce est relativement plus important dans U2.

Dans les strates hautes de $R$. racemosa/harisonnii, la biomasse sèche des racines est de 83,3 tonnes à l'hectare dans U3 et de 76,6 dans U4. La biomasse sèche racinaire est donc plus importante dans U3. L'explication se trouverait dans la théorie de Imbert (1985) citée précédemment puisque la différence de masse sèche peut s'expliquer par la proportion des tiges de gros calibre qui comptent $11,36 \%$ des tiges dans U3 contre $7,84 \%$ des tiges dans U4 selon Ndour (2005). Au-delà de la fixation dans la vase, l'entrelacs des racines a pour effet de renforcer le facteur d'élancement des tiges de mangrove qui varie de 40,39 à 68,3\% dans la zone (Ndour, 2005). Notre raisonnement s'appuie sur la théorie de Rondeux (1993) selon laquelle, le facteur d'élancement doit être supérieur à $80 \%$ pour que le peuplement soit considéré stable sur la terre ferme. D'ailleurs, il serait impossible aux palétuviers de vivre dans ce milieu sans leurs multiples racines échasses qui favorisent l'équilibre et la stabilité de leurs peuplements. En dehors de cet aspect, une évaluation de la biomasse racinaire souterraine pourrait révéler à la suite d'un cumul (biomasse racines échasses +biomasse souterraine) des quantités largement plus élevées que celles des tiges et autres parties.

Entre autres résultats, la biomasse sèche des tiges de $R$. mangle est de 55 tonnes à l'hectare dans U1, de 44,89 dans U2, de 26 dans U3 et de 69,1 dans U4. Il apparaît, selon Guiral et al. (1999), que la biomasse des tiges de R. mangle aux États-Unis (98,2 tonnes à l'hectare) est plus importante que celles obtenues au Saloum. Il est à noter aussi que la biomasse sèche des tiges de $R$. racemosa/harisonnii dans les strates hautes est de 65,9 tonnes à l'hectare dans U3 et de 62,5 dans U4. Dans le cadre d'une analyse diachronique, le résultat obtenu dans U4 concordent dans une moindre mesure avec celui de Doyen et al. (1985) où la biomasse des tiges de $R$. racemosa était de 60,1 tonnes à l'hectare dans la même zone. Considérant la biomasse la plus élevée au Delta du Saloum (65,9 tonnes à l'hectare), il apparaît aussi que nos mangroves sont moins productives que celles de la Thaïlande où $R$. apiculata produit 159 tonnes à l'hectare, de la Malaisie (257,4 à 286,8 tonnes à l'hectare) et du Panama où $R$. brevistyla a produit 278,9 tonnes à l'hectare (Guiral et al., 1999 ; Diouf, 1996).

\section{REMERCIEMENTS}

L'Institut des Sciences de l'Environnement de l'Université Cheikh Anta Diop de Dakar remercie vivement la Faculté des Sciences Agronomiques de Gembloux (FUSAGx) et la Direction des Parcs Nationaux (DPN) du Sénégal pour leur appui financier, logistique et institutionnel au cours de la collecte des données ayant abouti au présent article. Les auteurs dédient une mention spéciale au pilote de barque Monsieur El hadji Faye pour son exemplarité dans le travail.

\section{REFERENCES}

Baillon F. 1988. Le complexe hydro côtier du Sine-Saloum. Synthèse des paramètres écologiques et socio-économiques de la zone Nord du Delta. ORSTOM: Dakar ; 62.

Cormier-Salem MC. 1999. Des littoraux aux limites mouvantes. In Rivières $d u$ Sud. Sociétés et Mangroves Ouest Africaines (vol. 1). Edition IRD : Paris ; 17-30.

Dacosta H. 1992. Variabilité des précipitations sur le bassin versant du Saloum. In Gestion des ressources Côtières et Littorales du Sénégal. Actes de l'atelier de Gorée, pp. 87-103.

Diouf PS. 1996. Les peuplements de poissons des milieux estuariens de l'Afrique de l'Ouest : l'exemple de l'estuaire hyperhalin du Sine Saloum. Thèse de doctorat, Université de Montpollier II, $267 \mathrm{p}$. 
Doyen A, Agboba C. 1985. La mangrove à usages multiples de l'estuaire du Saloum (Sénégal), Master, Gembloux Agro-Bio Tech, ULg, Belgique, 145 p.

FAO. 1994. Les relations trophiques dans les écosystèmes de mangrove. Extrait de mangrove forest management guidelines. FAO Forestry Paper No. 117. Rome. http//www.fao.org/forestry mangrove.

Faye MN, Kaly JL, Diallo A, Guissé A. 2008. Influence des facteurs édaphiques sur la biomasse foliaire de deux espèces de palétuviers (Rhizophora sp., Avicennia africana ) dans la Réserve de Biosphère du Delta du Saloum, Sénégal. Journal des Sciences et Technologies, 7: 35-42.

Guiral D, Albaret JJ, Baran E, Bertrand FB, Loeuff LP, Montoroi JP, Sow M. 1999. Les écosystèmes de mangrove. In Rivières du Sud, Sociétés et Mangroves Ouest Africaines (vol.1). Edition IRD : Paris ; 416p.

ICA. 1989. Carte au 1/50 000 de la République du Sénégal. Japan International Coopération Agency et Gouvernement du Sénégal. Edit. Coupures de Sokone et de Thiès : Dakar.

Marius C. 1984. Contribution à l'étude des Mangroves du Sénégal et de la GambieEcologie-Pédologie-Géochimie. Mise en Valeur et Aménagement. ORSTOM : Paris ; 309 p.
Ndour N. 2005. Caractérisation et étude de la dynamique des peuplements de mangrove du Delta du Saloum (République du Sénégal). Dakar, Faculté des Sciences et techniques, Thèse de troisième cycle, Université Cheikh Anta Diop, 180 p.

Ndour N, Sambou B, Dieng SD. 2013. Production de litière de la mangrove du Parc National du Delta du Saloum (Sénégal). Int. J. Biol. Chim. Sci., 7(6): 2349-2369.

Ndour N, Dieng SD, Fall M. 2012. Rôles des mangroves, modes et perspectives de gestion au Delta du Saloum (Sénégal). Rev VertigO [en ligne], 11(3): 16p.

Rajendran N, Kathiresan K. 2007. Microbial flora associated with submerged mangrove leaf litter in India. Rev. Biol. Trop., 555(2): 393-400.

Rondeux J. 1993. La Mesure des Arbres et des Peuplements Forestiers. Edition Presses Agronomiques de Gembloux : Gembloux ; 521.

Wilmart G. 1996. Potentialités de développement d'une gestion intégrée pour les écosystèmes de mangrove. Aspect écologie et gestion, Mémoire de maîtrise; Université de Liège, Faculté des Sciences, 73p. 